\title{
Mechanical Aspects in Interferometric Gravity Wave Detectors
}

\author{
A. Rüdiger and colleagues of GEO ${ }^{1}$ \\ Max-Planck-Institut für Quantenoptik, D-8046 Garching, Germany \\ ${ }^{1}$ Names and affiliations of all authors are given at the end of the \\ paper
}

\begin{abstract}
In order to measure the tiny effects of gravitational waves, strains in space (i.e. relative changes in distance) of as little as $10^{-21}$ or even less have to be detected, at frequencies ranging from $100 \mathrm{~Hz}$ to several $\mathrm{kHz}$.

Large laser interferometers are the most promising approach to reach such extreme sensitivities. This 'straightforward' road is, however, obstructed by a multitude of effects that cause (or fake) such fluctuations in distance. Among these are seismic motions, thermal vibrations of optical components, pressure fluctuations of the residual gas in the vacuum tubes, and fundamental effects such as Heisenberg's uncertainty relation.

What all of these noise sources have in common is that their effects can be reduced by the choice of sufficiently large arm lengths. This is what dictates the (very expensive) choice of arm lengths of 3 to $4 \mathrm{~km}$ in the currently proposed gravitational wave detectors (USA, D-GB, F-I, AUS, JAP).
\end{abstract}

\section{Introduction}

\subsection{Objective of talk}

This is the third in our series of four talks on laser interferometric gravitational wave detection. The other talks are presented by G. Schäfer [1], K. Danzmann [2], and W. Winkler [3].

This talk will address the limitations in sensitivity imposed by mechanical noise of various kinds. It will discuss the origin of these noise sources and the methods to reduce them or suppress their effects. In particular, it will make clear why the long arm lengths envisaged are an absolute necessity. 


\subsection{Layout of talk}

The talk will be divided into three parts. A first part will set the scene, briefly recapitulating astrophysical background, proposals, basic parameters, appropriate representation of noise, and it will introduce our noise "yardstick", the shot noise.

The second part will treat what can be termed the "internal noise" sources, of rather fundamental nature: Heisenberg's uncertainty relation, thermal vibrations, and index fluctuations of the residual gas in thermal equilibrium.

And only then will the third part discuss the mechanical noise that one would normally think of first: seismic vibrations, as an example of "external" noise sources, and the ways to cope with them.

This sequence was chosen to be able to cover the very important topic of seismic isolation more broadly, and to be able to include a series of investigations that had just recently been performed.

\section{Setting the scene}

\subsection{Astrophysical Background}

The talk of G. Schäfer treated the most likely sources of gravitational radiation, and again recalled how extremely small their strain amplitudes, $h$, are. Let us just repeat in a few words the conclusions about the two main candidates for detection: supernovae and coalescing binaries. One should, however, keep in mind that each new observational window on the universe has brought totally unexpected discoveries, and we expect that the same will come true also in gravitational wave astronomy.

\subsubsection{Supernovae}

Burst sources such as supernovae, are rare events, and to wait for one of them to occur in our own galaxy, the Milky Way, may take a normal physicist's working life. For a higher rate of events, perhaps a few per month, we would have to look as far out as the Virgo cluster, to a distance of more than $10 \mathrm{Mpc}$.

Carefully performed ab initio calculations of the collapse processes [4] reveal that a rotationally symmetric collapse (at a distance of $10 \mathrm{Mpc}$ ) cannot be expected to produce peak strain amplitudes of more than $10^{-22}$. Calculations of similar reliability have not yet been performed for non-axisymmetric scenarios, but it is hoped that such cases might have up to an order of magnitude higher signals.

We conclude, therefore, that for burst sources a design sensitivity of $10^{-21}$ is the absolute minimum requirement, and clearly an upgrade to $10^{-22}$ 
must remain a long term goal. The typical frequency range for.burst sources is the region from a few hundred hertz up to a few $\mathrm{kHz}$.

\subsubsection{Coalescing binaries}

The other most likely source of gravitational radiation is the spiralling together of close binaries composed of highly condensed partners: neutron stars or - even more efficient - black holes.

The rate at which such events are to be expected is somewhat controversial; the statistics of such binaries is still very sparse. The general opinion is, however, that the rate is so low that a coverage of the universe even deeper into space than the Virgo cluster is required, perhaps as far as $100 \mathrm{Mpc}$.

The signal of such coalescing binaries would consist of a quasi-continuous wave of slowly rising frequency (a 'chirp'), and also of slowly rising amplitude. The signals are difficult to detect until they come into the range of, say, $100 \mathrm{~Hz}$. Within a few seconds, the evolving coalescence will reach frequencies of $200 \mathrm{~Hz}$, and then only fractions of a second until final splash-down.

A sensitivity of $10^{-22}$ (already taking into account the longer observation time) is the design goal for such coalescing binaries, and being able to measure down to, say, $100 \mathrm{~Hz}$ becomes even more important here.

\subsection{The proposals}

The presentation of $\mathrm{K}$. Danzmann gave an overview of the proposals made worldwide for building such laser-interferometric gravitational wave detectors of sufficient sensitivity. Even though some of the design details may differ between the three most advanced designs (GEO [5], LIGO [6], VIRGO [7]), there are some features that are very similar.

The most notable (and noticeable) common feature is the proposed length of the interferometer arms: $3 \mathrm{~km}$ each in VIRGO and GEO, $4 \mathrm{~km}$ in the wealthier and less populated United States (LIGO), and again $3 \mathrm{~km}$ in the Australian design [8] and the more recent Japanese concept [9].

This important design parameter, the arm length $\ell$, turns out to be the major cost factor; the cost of civil engineering and of the vacuum system is approximately proportional to the length $\ell$, and they make up close to $70 \%$ of the total cost. Thus, a reduction in arm length would cut down the detector cost considerably.

There have been suggestions from various researchers on how one could build interferometric gravitational wave detectors having much smaller dimensions, perhaps even of 'table top' size. One main objective of my talk is to state the physical facts that rule out such possibilities. The choice of arm lengths in the order of $3 \mathrm{~km}$ is not a reckless use of taxpayers' money, nor an attempt to build impressive monuments for posterity, but rather it is governed by physical necessities, if the dream of a gravitational wave astronomy is to become true. 
The suggested solutions of smaller sized detectors usually concentrated on how one can (or hopes to) defeat one particular noise effect, but then typically disregarded the other noise sources that also dictate the choice of long (km-sized) arms.

\subsection{Basic parameters}

In this subsection we will define some of the variables that will be used repeatedly in the sections to come, and give their typical range of values.

Of particular concern will be the arm length $\ell$, i.e. the geometric distance between the mirrors in each of the interferometer arms. When we discuss optical delay lines as proposed in the GEO project, the total optical path $L$ is given by multiplying $\ell$ with the number of passes, $N$ :

$$
L=N \ell \text {. }
$$

In GEO, in order to obtain light travel times $\tau=L / c$ that are appropriate for $\mathrm{kHz}$ signals $(\tau=0.3 \mathrm{sec})$, the total path $L$ needs to be in the order of $100 \mathrm{~km}$, so with $\ell=3 \mathrm{~km}$ we would need $N \approx 30$ passes in the delay line.

In LIGO and VIRGO, long light storage times are realized with FabryPerot cavities. The sensitivity with which gravitational wave signals can be detected is determined by the phase sensitivity, $\mathrm{d} \Phi / \mathrm{d} \ell$, and we see the finesse $\mathcal{F}$ play a similar rôle as the number of passes, $N$, in the delay line.

More recent ideas, such as the concept of "signal recycling" [10], make the distinction between the delay line scheme and the Fabry-Perot scheme less pronounced.

The various noise sources will be described with the specific GEO configuration in mind, but most of these are easily extrapolated to the configurations of LIGO and VIRGO. As will be seen, many of the effects can be discussed without having to make very specific assumptions about the particular design.

\subsection{Noise Representation}

The noise types to be treated here are all broadband, and of stochastic nature. A stochastic noise variable $v(t)$, of, say, dimension in meters, is then best represented by the spectral density (of the square) of the fluctuating variable, and it has become customary to give the linear spectral density denoted by

$$
\widetilde{v}(f), \quad \text { of dimension }[\mathrm{m} / \sqrt{\mathrm{Hz}}]
$$

such that the rms value in a given frequency band $\Delta f=f_{u}-f_{l}$ is given by

$$
v_{\mathrm{rms}}=\left(\int_{f_{t}}^{f_{u}} \widetilde{v}^{2}(f) \mathrm{d} f\right)^{\frac{1}{2}}, \quad \text { again of dimension }[\mathrm{m}] .
$$


It is important to keep in mind that the goal is to detect gravitational radiation in a frequency range that does not necessarily extend to very low frequencies.

In most cases in the following, we will assume a relatively large band width $\Delta f$ of the interferometer, i.e. on the order of the median frequency $f$ for which the interferometer is optimized. When a choice of $\Delta f$ has been made, the sensitivity obtainable can be expressed as a function of the design frequency $f$. It is this type of representation that is chosen for plotting the noise contributions in Figure 1, where a bandwidth of $\Delta f=f / 2$ is assumed throughout. The figure, taken from [5], also shows a rough indication of the magnitude of the expected signals.

\subsection{Shot noise - the limit?}

As is well known, the existence of shot noise gives rise to a very fundamental limit in sensitivity. The traditional representation is that shot noise fakes a fluctuation in phase of the measured output signal, or, in other words, an apparent fluctuation in total path length difference, $\widetilde{\delta L}$, as expressed in Equation (11) of W. Winkler's contribution [3]. When, as we will do here throughout, we express the noise as the attainable strain $h$, we find for the shot noise

$$
h_{\mathrm{SN}} \approx 2.4 \times 10^{-21}\left[\frac{\epsilon I_{0}}{50 \mathrm{~W}}\right]^{-1 / 2}\left[\frac{L}{100 \mathrm{~km}}\right]^{-1}\left[\frac{f}{1 \mathrm{kHz}}\right]^{3 / 2},
$$

where $\epsilon$ is the quantum efficiency of the detector, $I_{0}$ is the laser output power, and $f$ is the center frequency of the burst. It is noteworthy that this limit does not depend on the choice of the arm length $\ell$, but rather on the total path length $L=N \ell$, regardless of how this is realized by the two factors $N$ and $\ell$.

In W. Winkler's contribution, the consequences of shot noise have been made quite clear. Even for obtaining our more modest goal of $h<10^{-21}$ at $1 \mathrm{kHz}$, a light power of close to $1 \mathrm{~kW}$ would be required (of highly stabilized, single mode light); and for the eventual goal of $h<10^{-22}$ a truly prohibitive value of almost $100 \mathrm{~kW}$. No light source (laser) of such high power, which also satisfies all the other requirements, is anywhere in sight.

Fortunately, the light power inside the interferometer can be enhanced considerably by what is known as power recycling. The interferometer output is measured in the dark fringe of the interference. If for the moment we neglect the non-zero interferometer minimum, all light that is not lost due to the finite reflectivity loss, $(1-R)$, will be available for recycling (re-injecting) into the interferometer. The longer the arm length $\ell$, the fewer passes $N$ in the arms are required. This reduces the light loss due to the mirrors, and thus allows better power recycling. In this way, the strain sensitivity might reach best values of as little as 


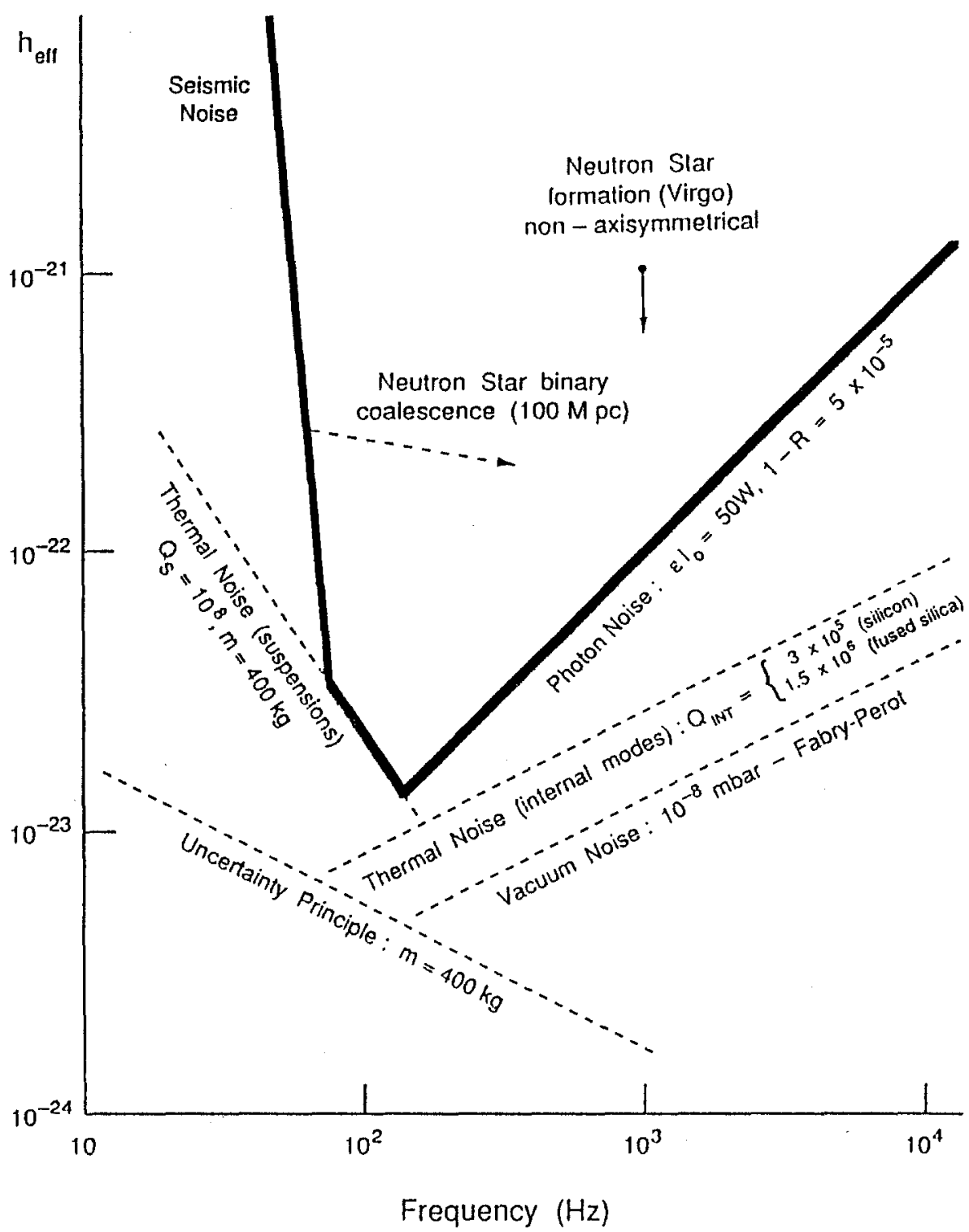

Fig. 1. This figure, taken from the German-British proposal [5], compares the strengths of two typical burst sources with the noise limitations imposed by the most prominent noise sources. For signals that allow observation over several oscillation periods, the effective amplitude $h_{\text {eff }}$ is approximately $h \sqrt{n / 2}$, where $h$ is the true amplitude and $n$ is the number of cycles of the waveform over which the signal can be integrated. 


$$
h_{\mathrm{SN}} \approx 10^{-22}\left[\frac{f}{1 \mathrm{kHz}}\right]\left[\frac{\epsilon I_{o}}{50 \mathrm{~W}}\right]^{-1 / 2}\left[\frac{1-R}{5 \times 10^{-5}}\right]^{1 / 2}\left[\frac{\ell}{3 \mathrm{~km}}\right]^{-1 / 2},
$$

and we see that the sensitivity $h_{\mathrm{SN}}$ improves (i.e.: drops) with the square root of the arm length $\ell$.

So, even before we get to the actual mechanical noise sources, we find that our "measuring stick", the shot noise limit, is dependent on the arm length $\ell$. In Figure 1, the heavy line denoted 'Photon Noise' assumes the design length of $\ell=3 \mathrm{~km}$. The sensitivity would deteriorate (the line would move upward) if arm lengths shorter than $3 \mathrm{~km}$ were chosen, jeopardizing the detection of such events as those indicated in Figure 1: supernovae and coalescing binaries.

\subsubsection{Squeezed light}

A way around the shot noise limitation could be found if non-classical states of light were to be used in the interferometer. Caves [11] pointed out that the photon counting noise in an ideal interferometer can be interpreted as stemming from the ground state vacuum fluctuations entering through the unused input port of the interferometer.

If one succeeded in replacing them by a specially prepared light of particularly small phase fluctuations, the photon counting noise could be reduced considerably. Such squeezed states of light have successfully been generated, but their usage in gravitational wave interferometers is still not in sight. Furthermore, the gain in signal-to-noise ratio will be very limited, as is pointed out in the contribution of W. Winkler [3].

\section{Internal Mechanical Noise}

\subsection{The Heisenberg Uncertainty Principle}

The indeterminacy in the simultaneous measurement of the position $x$ and the associated momentum $p_{x}$, as expressed in Heisenberg's uncertainty relation

$$
\Delta x \Delta p_{x} \geq \hbar / 2,
$$

gives a lower limit down to which a measurement of the current mirror displacement $\delta \ell$ is possible. One easily derives a (squared) spectral density

$$
\widetilde{h}^{2} \approx \frac{8 \hbar}{m \omega^{2} \ell^{2}}
$$

and, again with $\Delta f=f / 2$, we arrive at a sensitivity as shown in Figure 1 by the dotted line marked 'uncertainty principle'. From (7) we see that the linear sensitivity limitation is inversely proportional to the arm length $\ell$. 
The straight line is still safely below the heavy polygon that, as we will see, determines the sensitivity limitation of the GEO design. This is reassuring, and it is one of the great advantages of the interferometer detector over the resonant bars. Unlike in resonant bars (when we want to achieve even the more modest goal of $10^{-21}$ ), there is no necessity here to resort to such hard-to-realize schemes as "quantum non-demolition" or "back-actionevading" ... unless one wants to cut down on arm length. We clearly have here another good argument for $\mathrm{km}$ sized arms.

\subsection{Thermal Noise}

But things become worse if we consider yet other noise contributions. Let us take, as another important example, and also a very fundamental one, thermal vibrations. This example, too, has the advantage that its discussion needs no assumptions about the actual experimental implementation.

\subsubsection{Internal thermal motion of mirrors}

The thermal motion in the test masses introduces a vibration of the mirror surfaces that - for each relevant mode - can be described by a simple harmonic oscillator, of resonant frequency $f_{\mathrm{o}}=\omega_{\mathrm{o}} /(2 \pi)$. The damping is normally assumed to be proportional to velocity, and can be expressed by the quality factor $Q$. The (linear) spectral density of these motions can be written as

$$
\tilde{\mathscr{E}}(f)=\left(\frac{4 k T}{M \omega_{0}^{3}}\right)^{\frac{1}{2}} \cdot\left[Q\left(1-\left(\frac{\omega}{\omega_{\mathrm{o}}}\right)^{2}\right)^{2}+\frac{1}{Q}\left(\frac{\omega}{\omega_{\mathrm{o}}}\right)^{2}\right]^{-\frac{1}{2}},
$$

with a frequency dependence of the square bracket as shown in Figure 2. It is obvious that we do not want the resonant peak at $f_{0}$ to occur inside our frequency window of interest, since such a peak would have noise signals that are many orders of magnitude above the signal we want to measure.

We have to make sure that all resonant modes of the mirror substrate are well above our signal-frequency window. We then have to consider only the unavoidable low-frequency tails of theses modes, each of which has a white noise with a spectral density of

$$
\widetilde{\delta}(f)=\left(\frac{4 k T}{M Q \omega_{0}^{3}}\right)^{\frac{1}{2}}=\left(\frac{4 k T}{\pi^{3} \rho v_{\mathrm{s}}^{3} Q}\right)^{\frac{1}{2}} .
$$

Even with favorable assumptions, $M=400 \mathrm{~kg}$, a high mechanical $Q$ of $10^{6}$ (silicon), a resonant frequency of $f_{0}=3 \mathrm{kHz}$, and a bandwidth of $\Delta f=1 \mathrm{kHz}$, we get a $\delta l$ of $10^{-19} \mathrm{~m}$. Considering the number of mirrors and relevant modes involved, a total thermal motion of $10^{-18} \mathrm{~m}$ seems a. 


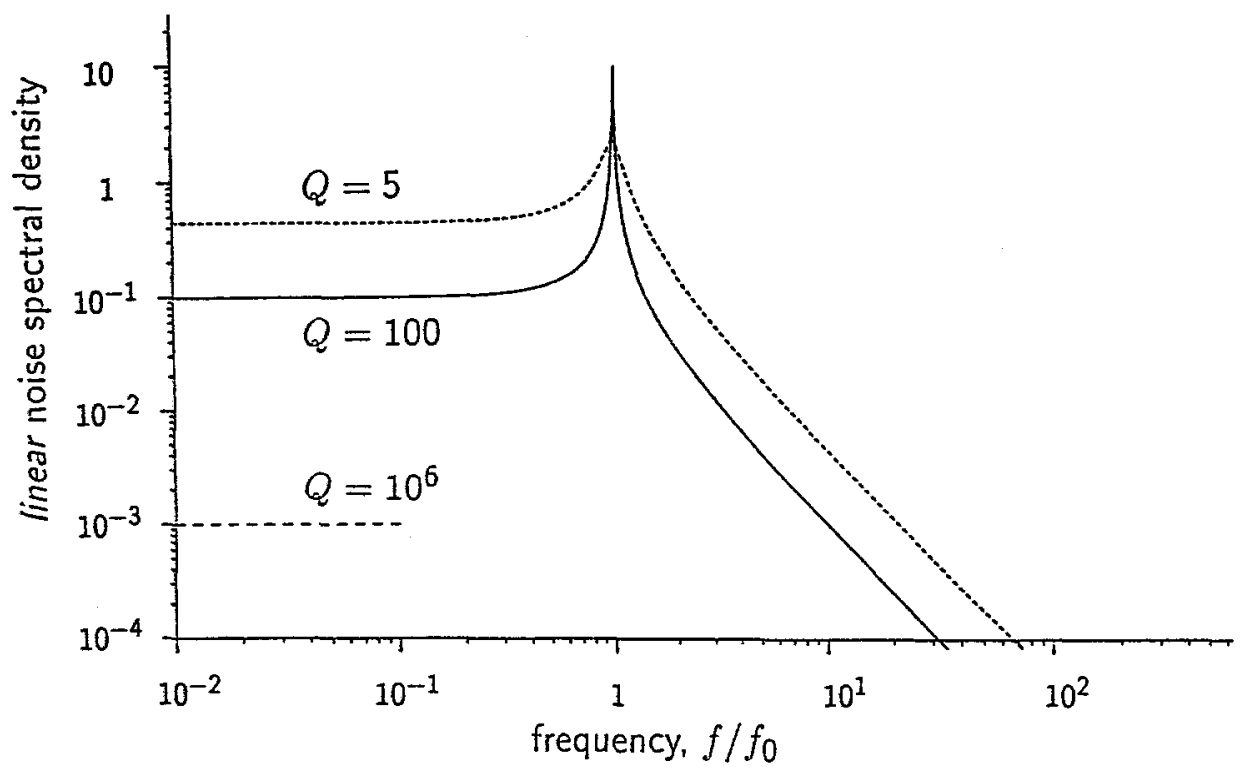

Fig. 2. Linear thermal noise spectral densities of oscillators with identical resonant frequency $f_{0}$, but different quality factors $Q$. For the extremely high $Q$ of $10^{6}$, only the level of the subresonant white-noise tail is indicated.

reasonably safe upper estimate, and from this we deduce that we can get near the required goal of $\delta / \ell=10^{-21}$ only with arm lengths $\ell$ of a few kilometers, say, $3 \mathrm{~km}$.

In order to avoid the relatively low frequencies of the bending modes, the substrate aspect ratios (thickness over radius) will be chosen close to unity. It is interesting to note that the white noise of (9) then becomes independent of the size of the mirror substrate, the length dependencies in $M$ and $\omega^{3}$ cancel each other.

Expressing the noise as a (squared) strain spectral density,

$$
\tilde{h}^{2} \approx \frac{16 k T}{\pi^{3} \rho v_{\mathbf{s}}^{3} Q \ell^{2}}
$$

and again using the bandwidth $\Delta f=f / 2$ and a safety margin of 2.5 to take into account the presence of several modes, we find a sensitivity that is shown in Figure 1 as the dotted line marked 'Thermal Noise (internal modes)'. We can clearly see that for the materials assumed one can just barely keep below the shot noise limit at low frequencies, whereas we have a rather comfortable margin at higher frequencies. 


\subsubsection{Choice of materials}

A look at (10) gives us some indication about what characteristics of substrate materials to look for. The velocity of sound, $v_{\mathbf{s}}$, enters very strongly, more so than the specific weight $\rho$. Many substrate materials have much better figures of $\rho v_{s}^{3}$ than fused silica, and at the same time also better quality factors $Q$. Silicon and sapphire (both already available in rather large single crystal ingots), would both have good characteristics, even better (because of sound velocities $v_{\mathbf{s}}$ above $10 \mathrm{~km} / \mathrm{sec}$ ) would be beryllium (toxic!), and - better yet - beryllia and diamond. Beryllia crystals have so far been a militarily classified material, so not very much is known about large specimens. Diamond is being grown by novel processes now, but so far only in thin layers (up to $1 \mathrm{~mm}$ ). These alternative materials also have advantages in their thermal properties which make them less susceptible to problems associated with heating due to the light beam, as discussed ir. W. Winkler's contribution [3]. Only the future can tell whether these ideal materials will become available in the sizes required.

\subsubsection{Table top interferometer?}

What if we wanted to attain the same sensitivity with a short interferometer of, say, $1 \mathrm{~m}$ in length ? Let us look at the second expression in Equation (9). Not very much more than a factor of ten can be gained via the factor $\rho v_{s}^{3}$, so for more drastic improvements we would have only the parameters $T$ and $Q$ to play with, both entering under the square root.

We could cool the substrate, by six powers of ten, to $T=300 \mu \mathrm{K}$. This certainly is not an attractive solution: One would have to make sure that the vacuum system as a whole is even colder than the mirrors, unless we want our top quality mirror surfaces to trap the residual gases. But worse yet, this scheme is impossible: Even assuming mirror coatings with absorption losses of only $0.1 \mathrm{ppm}$, i.e. ten times less than the best ones made today, the dissipation on the mirror surfaces could be radiated away only at substrate temperatures of, say, $10 \mathrm{~K}$. Trying to remove the dissipated light power by heat conduction is hard to reconcile with the high demand on seismic isolation.

We could hope for materials with higher $Q$. The fused silica which we consider for our mirrors is already a material of very high $Q\left(Q \approx 10^{5}\right)$, but some better materials are known, particularly if one cools them down to or below liquid helium temperatures. Pure single crystal sapphire is known to have an extremely high $Q$, perhaps up to something like $10^{9}$. So with the combined efforts (and huge expenses) of sapphire end masses and cryogenics, one might marginally get to the required 6 powers of ten. But with that we have defeated only one enemy, although admittedly a very prominent one. 


\subsubsection{Thermal motion of pendulums}

A very different regime of the resonant curve of Figure 2 applies when we consider the thermal noise of the pendulum suspension. Here the resonant frequency of the 'pendulation mode' is way below our frequency range of measurement, and only the high-frequency tail enters. The sensitivity limit due to the suspension noise is then determined by the noise spectral density

$$
\tilde{h}^{2} \approx \frac{16 k T \omega_{0}}{m Q_{\mathrm{S}} \omega^{4} \ell^{2}}
$$

At higher frequencies, its contributions are negligible, but not so at frequencies around $100 \mathrm{~Hz}$. This is seen, again with our GEO specifications in mind, from the dotted (and partly heavy) line marked 'Thermal Noise (suspension)'. For this line, the very favorable assumption of a pendulation $Q$ of as high as $10^{8}$ was assumed.

Such a high $Q$ is not only difficult to obtain, it is also extremely difficult to measure or verify. The $Q$ gives the number of oscillations after which the amplitude of the motion has fallen by a factor $1 / e$. At a period in the order of 1 second, $10^{8}$ oscillations will take about 3 years. Not only would a measurement be stretching the patience of the experimenter, also any seismic influences that might add to (or subtract from) the present state of oscillation must be strictly avoided.

Values of $Q$ up to $10^{7}$ have been measured [12]. Although some groups have proposed to measure the decay more directly (by going to yet lower frequencies) the $Q$ assumed here is not a well-established figure, but rather one that can be derived by physical arguments, considering the heavy end mass, and the very tiny area (near the top of the suspension wire) where any dissipation is to be expected.

As we see from Figure 1, this suspension noise already affects the attainable sensitivity, so clearly any reduction in arm length will increase the noise limit, with the inverse of $\ell$. No reduction can be afforded, particularly not if detection is intended to reach into the frequency range of $100 \mathrm{~Hz}$. Particularly for the VIRGO project, with its declared aim of measuring down to frequencies below $100 \mathrm{~Hz}$, this suspension noise would pose a serious problem.

\subsection{5 'Imaginary spring constant' damping}

Recently, the question of how best to represent mechanical damping has been looked at more closely by P. Saulson [13] and others. These researchers have proposed (and observed) a different damping law, and it is not unlikely that this law also applies to the mechanical systems we are dealing with here. Rather than describing the damping by a velocity-proportional term in the differential equation, it is described (in the frequency domain) by 
an imaginary (and possibly frequency dependent) component in the spring constant $k$ :

$$
k=k_{\mathrm{o}}[1+i \phi(\omega)] .
$$

Such a modified friction law will not influence the characteristics of the seismic isolation very substantially, but it does have serious implications on thermal noise. In the standard damping model the thermal displacement noise of the mass, as shown in Figure 2, has the same shape as the transfer function of the harmonic oscillator; this is because the oscillator is driven by a 'white' random force. This case is plotted as the solid line in Figure 3.

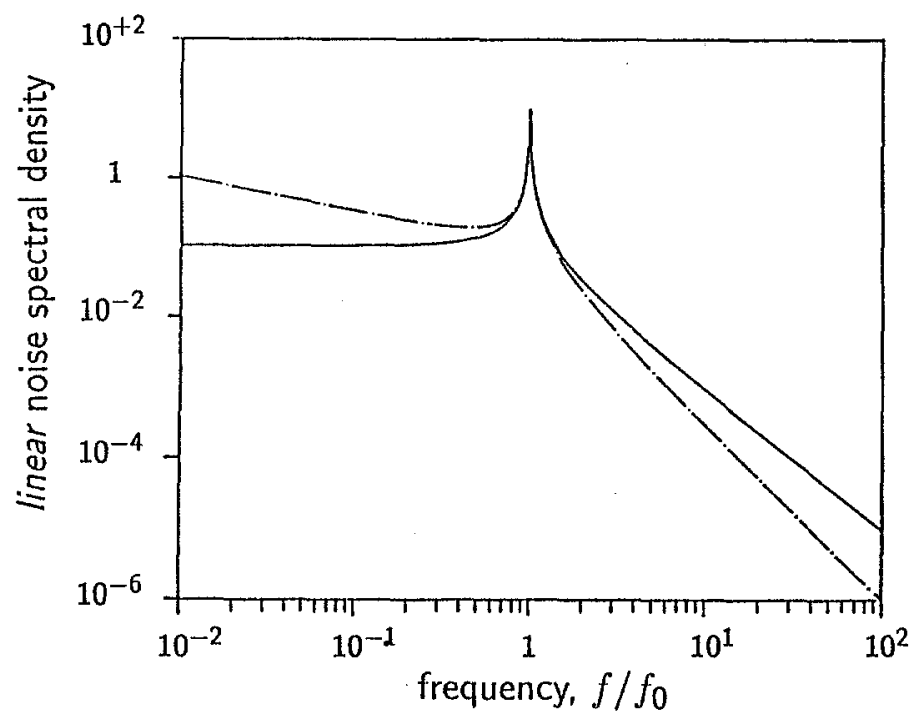

Fig. 3. Thermal noise spectrum of a harmonic oscillator: for velocity-proportional damping (solid curve) and for 'imaginary spring constant' damping (dashed curve), $Q=100$, after Saulson [13]. Linear spectral densities as in Fig. 2.

In the case of the 'imaginary spring constant' damping, however, the thermal driving force turns out to be frequency dependent, and the spectral density of the thermal noise rises towards low frequencies, but falls off more rapidly above resonance [13]. This is shown as the dotted line in Figure 3.

If this modified damping law turns out to be a better description, then the subresonant tails of the substrate vibrations will become a greater problem. In Figure 1, this would make the right-hand line named 'Thermal noise (internal modes)' shallower, and higher at the low-frequency side. The line could then possibly slightly exceed the shot noise curve in the deep trough where photon noise crosses the suspension noise.

If this loss mechanism applies to the pendulum suspension, the 'aboveresonant' roll-off in Figure 1 will become even steeper, relaxing the requirements for the suspension's thermal noise. This fact would help the VIRGO project at low frequencies. 


\subsection{Refractive index fluctuations in the residual gas}

The light will travel between the mirrors in highly evacuated tubes, but local changes in the number of molecules remaining in the light path will lead to fluctuations in the refractive index. This changes the optical path between the mirrors without actually changing their positions.

Sudden changes (only these would cause signals in our frequency range of interest) could be caused by local gas eruptions from the tube walls, and even more so from the surfaces of the pumps, particularly getter pumps. A recent research program at PTB in Berlin is making headway in investigating these phenomena at the pressures and time scales that are of interest here.

An estimate of the lower bound of the refractive index noise can be made by treating the residual gas as being in thermodynamic equilibrium. Each single molecule traversing the beam of width $2 w$ will retard the light phase by an amount that depends on the relative field strength at its location. By averaging over all possible positions and flight directions (and thus interaction times), assuming a Maxwell distribution of the molecule velocity $v$, and weighting with the (gaussian) field strength, one can calculate the autocorrelation function $R(\tau)$ of the effect of these atoms. It was a pleasant surprise that the analytical treatment, after many integrations of higher transcendental functions (Dawson's integral, modified Bessel functions) of complicated arguments, finally led to the simplest autocorrelation function imaginable, a Lorentzian of the form

$$
R(\tau)=\frac{1}{1+\left(\tau / t_{R}\right)^{2}}
$$

where the mean interaction time $t_{\mathrm{R}}$ is given by $t_{\mathrm{R}}=\sqrt{2} w / v_{\mathrm{m}}$ and where the thermal velocity $v_{\mathrm{m}}$ stands for the "most probable velocity" $v_{\mathrm{m}}=\sqrt{2 k T / m}$ in the Maxwell distribution

$$
\frac{4}{\sqrt{\pi}} \frac{v}{v_{\mathrm{m}}} \mathrm{e}^{-\left(v / v_{\mathrm{m}}\right)^{2}}
$$

The autocorrelation function $R(\tau)$ leads to a (single-sided) power spectral density equalling twice the Fourier transform,

$$
S(f):=2 \int_{-\infty}^{\infty} R(\tau) \mathrm{e}^{i \omega \tau} \mathrm{d} \tau=\frac{1}{f_{c}} \cdot \mathrm{e}^{-f / f_{\mathrm{c}}} .
$$

For frequencies well below the 'cut-off frequency' $f_{c}=1 /\left(2 \pi t_{R}\right)$ the 'vacuum noise' (15) is almost frequency independent (white). With the usual GEO characteristics, we find the (squared) strain noise to be

$$
\widetilde{h}^{2} \approx \frac{2 \sqrt{2}\left(n_{0}-1\right)^{2}}{N_{0} v_{0} \cdot \ell \bar{w}}\left(\frac{p}{p_{0}}\right)
$$


where $n_{0}$ and $v_{0}$ are the refractive index and the mean velocity of the gas in question, and $N_{0}$ is the number of molecules per unit volume at standard pressure $p_{0}:\left(2.7 \times 10^{25}\right.$ molecules $\left./ \mathrm{m}^{3}\right)$.

The fluctuations (16) determine the maximum allowable gas pressure $p$ for a given strain sensitivity $h$. To be on the safe side, the vacuum specifications for GEO were laid down as: $<10^{-8} \mathrm{mbar}$ for hydrogen and $<10^{-9}$ mbar for heavier molecules, such as water and nitrogen. This leads to the line marked "Vacuum Noise" in Figure 1.

The beam width $2 w$ is a function of arm length $\ell$ and wavelength $\lambda$, $w \approx \sqrt{\ell \lambda / \pi}$, so in the end we find $\widetilde{h}$ to be proportional to $\ell^{-3 / 4}$, and for any arm length $\ell$ below $1 \mathrm{~km}$, the vacuum specifications would have to be tightened beyond what can be done at reasonable cost.

Thus, also the effect of index fluctuations gets reduced by the choice of a longer arm length, mainly due to the better 'averaging' over the longer beam length $\ell$ and the wider beam diameter $2 w$.

\section{Seismic Isolation}

The third part of this talk deals with the motions of the ground at the site of the interferometer, a noise that is generally termed 'seismic', even though it is not necessarily and exclusively of geophysical origin. Discussing the efforts required to suppress noise due to these seismic motions (see also N. Robertson [14]) will again bring to our attention how extremely small the gravitational wave effects are that we want to measure.

\subsection{Seismic noise}

\subsubsection{The frequency range}

We will have to cope with seismic noise over a wide frequency spectrum, a few $\mathrm{kHz}$ at the high end, and down to semidiurnal tidal deformation of the earth's crust, or even seasonal variations, at the low end.

Although the gravitational waves are to be measured in a rather limited frequency band only, from, say, $100 \mathrm{~Hz}$ to a few $\mathrm{kHz}$, it is nonetheless necessary to consider the effects due to high-amplitude motions (slow drifts) at the extremely low frequencies. They can make the interferometer deviate too far from its ideal point of operation.

It takes a wide spectrum of measuring devices to cover this vast frequency range: (piezo-type) accelerometers for frequencies from $\mathrm{kHz}$ downward to $10 \mathrm{~Hz}$; seismometers (mostly velocity-proportional, dip-coil) from $100 \mathrm{~Hz}$ down to $1 \mathrm{~Hz}$, in astatized seismometers down to $10^{-1} \mathrm{~Hz}$; and strain measurements (mechanical and laser-interferometric) between two measuring points down to seasonal and secular variations. 


\subsubsection{The model}

For the purposes of designing a seismic isolation system, it has become customary to model the seismic motion of the ground by a (linear) spectral density of the displacement

$$
\widetilde{x}(f)=10^{-7} \frac{\mathrm{m}}{\sqrt{\mathrm{Hz}}} \cdot\left[\frac{1 \mathrm{~Hz}}{f}\right]^{2},
$$

which describes the frequency dependence quite well over a wide frequency spectrum, certainly from $\mathrm{kHz}$ downward to, say, the microseismic frequencies at $\approx 10^{-1} \mathrm{~Hz}$. This equation represents a worst case motion at the sites being considered, at very quiet sites the amplitudes might be by a factor of ten lower.

\subsubsection{Site selection}

It is quite natural that in selecting a site for the experiment one will not want to pick a particularly noisy place. Man-made noise arising from traffic, industry, agriculture, etc. contributes most strongly in the frequency range from a few $\mathrm{Hz}$ up to, say, $100 \mathrm{~Hz}$. Sufficient distance (one to several $\mathrm{km}$ ) from busy roads, railroad tracks, heavy industry, mining, (and more than $10 \mathrm{~km}$ from military artillery ranges) must be guaranteed.

Coastal regions have strong ground noise contributions from the surf and swell of the sea. The microseismic phenomena, on the other hand, and in particular the 'microseismic storms', at frequencies of 0.1 to $0.15 \mathrm{~Hz}$ are believed to stem mostly from the swell of heavy sea, and to travel far into the mainland. There is no obvious way to escape from them, at least not inside Europe.

If one excludes the obviously unappropriate sites, the ground noise (in the range from $1 \mathrm{~Hz}$ to $100 \mathrm{~Hz}$ ) still shows a wide variation depending on the geological formation.

In an earlier series of measurements, Steinwachs [15] had established that the seismic noise increases monotonically with the height of loose rock or scree above bedrock. Furthermore, the man-made noise propagates mainly as a surface wave, and its amplitudes drop rapidly as one goes far enough (at least $10 \mathrm{~m}$, better $100 \mathrm{~m}$ ) below surface.

Such arguments have again raised the interest in below-ground sites, and particularly into tunnels driven into hard bedrock. With modern techniques, the additional cost over surface installations no longer seems prohibitive. Sites in (seismologically stable) mountain ranges in the German state of Niedersachsen are being considered, and recent seismic measurements [16] in abandoned mines there have established their excellent usefulness. 


\subsubsection{Isolation required}

The degree of isolation required depends on the design parameters of the detector: the sensitivity $h_{\mathrm{D}}$ aimed at, the design frequency $\bar{f}$, and the bandwidth $\Delta f$.

From the rather steep roll-off of the ground motion, Equation (17), and from the fact that all methods of isolation improve with frequency, we can expect that isolation at higher frequencies must be a relatively easy job.

Let us take as a first example a modest design sensitivity of $h_{\mathfrak{D}}=10^{-21}$ at $\bar{f}=1 \mathrm{kHz}, \Delta f=500 \mathrm{~Hz}$, and $\ell=3 \mathrm{~km}$. The rms ground motion in this band is about $x_{\mathrm{rms}}=2.5 \cdot 10^{-12} \mathrm{~m}$. With 4 mirrors involved in the measurement, we see that even for this case a suppression of the ground noise by 10 powers of ten is required to arrive at $h_{\mathrm{D}}=10^{-21}$.

For the more ambitious goal of $h_{\mathrm{D}}=10^{-22}$ at $100 \mathrm{~Hz}, \Delta f=100 \mathrm{~Hz}$, we find $x_{\mathrm{rms}}=1.6 \cdot 10^{-10}$, so we need a suppression by 13 powers of ten, which is much more difficult, particularly at these lower frequencies.

The next sections will give some examples of isolation methods with which one can hope to achieve these suppression values.

\subsection{Isolation by pendulums}

\subsubsection{Single pendulum suspension}

The simplest way to isolate a mirror from high-frequency ground motion is to suspend it as a pendulum by one or several thin wires. The method chosen at Garching was to hold the mirror by a thin steel wire sling, as shown schematically in Figure 4.

The damping is typically very low for such a suspension system, particularly if care is taken to avoid friction at the lift-off point of the wire from the mirror, as well as at the suspension point.

The typical frequency response of the mirror motion $\widetilde{z}(f)$ for a given 'ground' motion $\widetilde{x}(f)$ of the suspension point, the transfer function $\widetilde{H}(f)=$ $\widetilde{z}(f) / \widetilde{x}(f)$, is given by the simple resonant curve of the shape already shown in Figure 2. At frequencies well above the pendulum's resonant frequency $f_{\mathrm{P}}$, this transfer function (also called transmissibility) rolls off as $\left(f_{\mathrm{P}} / f\right)^{2}$.

For reasonable wire lengths $l_{\mathrm{p}}$, on the order of $1 \mathrm{~m}$, the resonant frequency is near

$$
f_{\mathrm{P}}=\frac{1}{2 \pi} \sqrt{\frac{g}{l_{\mathrm{P}}}} \approx 0.5 \mathrm{~Hz} .
$$

Although at $1 \mathrm{kHz}$ we then have a transmissibility of less than $10^{-6}$, this still falls short of even our modest goal of a reduction by $10^{-10}$. Much longer pendulums are not practicable, and they could never provide the missing four powers of 10 . 


\subsubsection{Wire resonances}

Another obstacle in reaching the desired suppression is the fact that the suspension wires are not massless and thus have their own resonances. The high amplitudes at these resonances are transformed (though reduced by the mass ratio $\mu / m$ of effective wire mass $\mu$ to mirror mass $m$ ) into motions of the mirrors.

The wire pendulum can be treated in close analogy to an electrical transmission line terminated with an inductance (to represent the inertial termination by the impedance $Z_{\mathrm{P}}=i \omega m$ of the pendulum mass $m$ ). The characteristic impedance $Z=\sqrt{m g \gamma}$ of the mechanical transmission line is given by the tensile force $m g$ on the wire and the linear mass density $\gamma$. The propagation constant $k=\omega / v_{\mathrm{tr}}$ is determined by the velocity $v_{\mathrm{tr}}=\sqrt{m g / \gamma}$ with which a transverse motion propagates along the wire.

As in an electrical transmission line, the displacement $x_{\mathrm{P}}$ at the termination (pendulum mass) is transformed to the front end (suspension point) via a transformation

$$
x_{0}=x_{\mathrm{P}}\left(\frac{Z_{\mathrm{P}}}{Z} i \sin k l+\cos k l\right),
$$

and one arrives at the transfer function

$$
H(f):=\frac{x_{\mathbf{P}}}{x_{0}}=\frac{1}{\cos k l-\frac{\omega m}{Z} \sin k l} .
$$

The gravest resonance $\omega_{\mathrm{P}}=\sqrt{g / l}$ (the pendulation mode) and the lowfrequency transfer function $H(f)=\left(1-\left(f / f_{\mathrm{P}}\right)^{2}\right)^{-1}$ are easily derived by expanding for $k l \ll 1$. All further resonances (the 'violin string' resonances $f_{n}$ ) can be found from the approximation $k l \approx n \pi$, leading to

$$
f_{n} \approx n \pi f_{\mathbf{P}} \sqrt{\frac{m}{\mu}},
$$

with $\mu=\gamma \cdot l$ the mass of the wire sling (two wires).

Figure 4 shows the suspension system used in the 1986 Garching prototype [17], and the theoretical and experimental transfer functions. For the values used ( $m=1.1 \mathrm{~kg}$, steel wire $0.1 \mathrm{~mm}$ in diameter), the mass ratio $m / \mu$ is about 12500 , and the wire resonances are in very good agreement with the measured peaks at multiples of $f_{1} \approx 212 \mathrm{~Hz}$. At these frequencies, the pendulum suspension not only loses its isolation feature, it even enhances the motion of the pendulum over that of the ground.

In between these resonances, the transfer function $H(f)$ provides an isolation that is at best

$$
H(f) \approx \frac{Z}{\omega m}=\frac{f_{\mathrm{P}}}{f} \sqrt{\frac{\mu}{m}}
$$




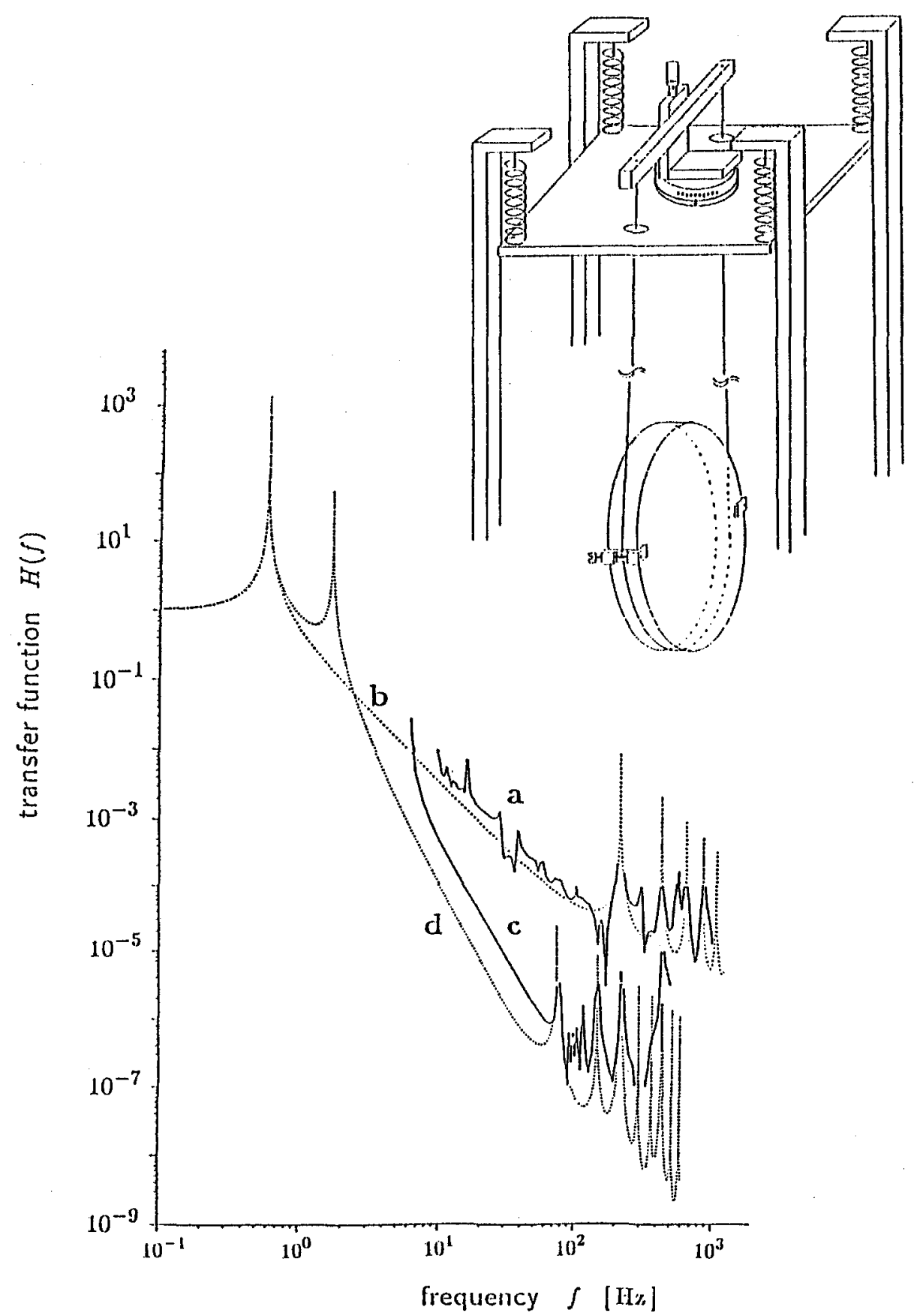

Fig. 4. Top: Schematic view of a (double) pendulum suspension, as in the Garching $30-\mathrm{m}$ prototype of 1986 . The upper stage, supported by four coil springs, carries translation and rotation stages for the suspension wire. The upper suspension is $0.10 \mathrm{~m}$ in length, the lower $0.72 \mathrm{~m}$.

Bottom: The curves $\mathbf{a}$ and $\mathbf{b}$ are the measured and calculated transfer functions for a one-stage pendulum, $\mathbf{c}$ and $\mathbf{d}$ for two stages. 
i.e. it rolls off only with $1 / f$. With the above values and at, say, $550 \mathrm{~Hz}$ one finds an isolation by about $10^{-5}$.

\subsubsection{Local control}

The high quality factor $Q$ of the pendulum has the undesirable consequence of a very high resonant peak (see Figure 2). Using a pendulum with increased friction (and thus lower $Q$ ) would not be an ideal remedy. For the mirror mass, a low $Q$ would be unacceptable from the thermal noise considerations of section 2.2.4. But also for an intermediate mass in a double pendulum system (see section 3.2.4), assuming simple velocity-proportional damping, the desirable feature of a roll-off with $\left(f_{\mathrm{P}} / f\right)^{2}$ is valid only up to a frequency $f_{Q} \approx Q f_{\mathrm{P}}$, from then on the further roll-off goes only with the inverse of $f$.

There is, however, a way to combine a shallow resonant peak with the desired $f^{-2}$ roll-off. This is a frequency-selective damping incorporated in what we call 'local control' servos $[18,17]$. This feed-back control measures the relative position of the mirror via a rather crude technique (a shadow sensor) and feeds back a low-passed control signal via coils that act on permanent magnets attached to the mirrors and/or the intermediate masses. The control currents are manipulated such that they damp the pendulum mode, but at higher frequencies leave the $f^{-2}$ roll-off untouched.

These coil-and-magnet controls, now widely used in several prototypes, serve also other purposes, e.g. in very-low frequency control and for optical alignment.

\subsubsection{Double pendulum}

We demonstrated that a single pendulum cannot provide sufficient suppression even to satisfy our less ambitious goal of $10^{-21}$ at $1 \mathrm{kHz}$. An obvious approach is to use multiple pendulums, in the simplest case a two-stage pendulum. This is what was implemented in the Garching 30-m prototype. A schematic view of this suspension scheme is shown in Figure 4. The simple "wireless" model would give a straight $\left(f_{\mathrm{P}} / f\right)^{4}$ roll-off. This would lead to a suppression by better than $10^{-13}$ at $1 \mathrm{kHz}$, but such values are not reached in a more realistic model.

The calculation of the transfer function including the wire resonances is a straightforward extension of that for the single pendulum. The comparison of the experimental and theoretical transmissibilities is given by the two lower curves in Figure 4. Again, rather good agreement is found, also in the kink in the roll-off, which (in the valleys between the resonances) is expected to go with $f^{-2}$.

The wire resonances limit the suppression that we can reach, and although the two-stage pendulum was found sufficient for the 30-m prototype, for the large projects a much better seismic isolation will be needed. 


\subsubsection{Vertical motion}

One severe draw-back of wire pendulums is that their vertical isolation is inferior to the horizontal one. This is easily understood when we consider the resonant frequency for the tensile vertical motion,

$$
f_{\mathrm{v}}=\frac{1}{2 \pi} \sqrt{\frac{g}{\Delta l}}
$$

where $\Delta l$ is the elongation of the wire length $l_{p}$ due to the weight of the suspended mirror, and when we compare this with the horizontal pendulation frequency of Equation (18). The elastic, reversible regime for most wire materials will not go much beyond strains $\Delta l / l_{\mathrm{P}}$ of $1 \%$. So the vertical resonant frequency $f_{\mathrm{v}}$ is at least one power of 10 higher than the horizontal resonance. Above this resonance, a single pendulum will be about two powers of ten inferior in its vertical isolation, a double pendulum four powers of 10 .

This shortcoming of the wire pendulum is, fortunately, not as dramatic as these numbers suggest, since the interferometer is in first approximation insensitive to mirror motions transverse to the optical axis.

There are, however, several mechanisms that may convert vertical motion into horizontal changes in mirror distance. A very fundamental one is the finite radius of the Earth. The vertical motions of the mirror spaced $3 \mathrm{~km}$ subtend an angle of $0.5 \mathrm{mrad}, 0.5 \cdot 10^{-3}$, and it is this factor by which vertical motions (pointing to the center of the Earth) are converted into longitudinal distance variations.

There are many structural features that can also transform vertical, or tilting, motions into horizontal ones. In all cases, the conversion factor will be quite small, typically perhaps in the order $10^{-2}$, but this just about compensates the inferior vertical isolation of a single pendulum stage. The vertical motions are not an unsolvable problem, but they must always be kept in mind in the design of the isolation system.

\subsubsection{Multiple pendulums, VIRGO}

A very ambitious scheme is being developed (and being tested) for the VIRGO project. The aim of this project is to be able to measure at even lower frequencies than $100 \mathrm{~Hz}$. This makes achieving a good seismic isolation even more important (as well as more difficult).

The mirror masses are suspended by a chain of seven pendulums in series, with a total height of about $7 \mathrm{~m}$, the total vacuum chamber towering $12 \mathrm{~m}$. The individual stages are made from air springs, and they have vertical resonant frequencies that come quite close to the ones of the horizontal ('pendulation') modes. This is an important advantage over wire pendulums. 
The roll-off with $f^{-14}$ has been verified over a limited frequency range, and very impressive transmissibilities have been measured. Only a very sensitive interferometric measurement will be able to establish how good the transfer function is at higher frequencies. This 7 -stage pendulum system is expected to come close to, but not yet quite reach, the very ambitious isolation specifications for VIRGO. The addition of an 'inverted pendulum' at the top of the 7 -stage pendulum seems to be provide the required additional isolation.

\subsection{Isolation via lead-and-rubber stacks}

\subsubsection{Stacks}

As we have seen, the double pendulum cannot sufficiently isolate the mirrors, even for the relaxed goals of $h \approx 10^{-21}$ at $1 \mathrm{kHz}$. There is, however, the possibility additionally to isolate the suspension point via stacks made up of alternating layers of heavy (e.g. lead) bricks and a soft, elastic material (e.g. rubber or elastomers). Such stacks have been used very successfully in the seismic isolation of resonant bar gravitational wave antennas where very impressive seismic isolation values have been achieved. Such stacks have been used in interferometer prototypes at Glasgow and later at Caltech, and are now being implemented in the Garching $30-\mathrm{m}$ prototype.

Stacks of such alternating layers have some features that are similar to those of the multiple pendulums. The more stages one uses, the steeper is the roll-off at frequencies sufficiently above the highest resonant mode of the stack. (This highest mode, incidentally, is the mode in which the heavy layers have alternating direction of motion).

\subsubsection{Damping of stacks}

Unlike the (multiple) pendulums, the stacks are typically systems of relatively high internal losses, i.e. of low $Q$. Values of $Q$ between 1 and 10 are typical. An immediate consequence of this low $Q$ is that the roll-off goes with $f^{-n}$, where $n$ is the number of stages, and not with $f^{-2 n}$ as in the case of multiple pendulums.

Although the steeper roll-off with $f^{-2 n}$ would be more desirable, it would not outweigh the advantage of having the elastic layers made out of a very lossy material. One could easily achieve the high compliance (the 'softness') for instance with metallic coil springs, but these would, at higher frequencies, have their own internal resonances, entirely ruining the stack's isolation characteristics. 


\subsubsection{RAL stacks}

A set of lead-and-rubber stacks was designed by Rutherford Appleton Laboratories, to be tested and used in an upgrade of the Garching 30-m prototype.

Boundary conditions in the design were the inner diameter $(1000 \mathrm{~mm})$ and the limited free headroom in the Garching vacuum tanks. A top plate (from which the double pendulum system is suspended) is supported by four stacks at the four corners. The stacks can have up to five stages and a total height of $86 \mathrm{~mm}$.

Each lead brick has a mass of $4 \mathrm{~kg}$. The rubber springs are cylindrical, $25 \mathrm{~mm}$ in diameter and $40 \mathrm{~mm}$ high (unloaded). There are four such rubber 'springs' in each layer between two lead bricks, at each of the four corner 'substacks'.

The bricks have cylindrical recesses of half the brick's thickness so that the bricks are supported in the plane of their center of mass. This is to avoid that tilting motions convert into horizontal displacements.

Measurements of the transfer function (the transmissibility) performed at RAL are shown as the solid curve of Figure 5. These measurements were made with vertical driving forces acting on the stack's bottom plate. The 'gravest' (i.e. the lowest) resonance, at about $6 \mathrm{~Hz}$, was below the frequency range covered at RAL. The total number of peaks (4) is identical with the number of stages.

The roll-off at frequencies above, say, $40 \mathrm{~Hz}$ is quite steep, and a suppression of $10^{-4}$ was reached at about $70 \mathrm{~Hz}$.

\subsubsection{Transmissibility of stacks}

Using a simple one-dimensional model of such stacks, assuming rigid metal bricks, with elastic, lossy rubber springs in between, one can derive a recursive scheme with which one can easily calculate the transmissibility for any given number of stages $(n)$. The stages need not be identical, and some dependence of the rubber compliance on the total load can also be incorporated.

The dotted line in Figure 5 shows the computer calculation of the transfer function, taking the mass $(4 \mathrm{~kg})$ and the specified vertical stiffness $(22 \mathrm{~N} / \mathrm{mm}$, or a compliance of $0.045 \mathrm{~mm} / \mathrm{N}$ for a single rubber spring). Only the loss factor, or the $Q$, was fitted, such that the height of the resonant peaks was similar to the measured data. Quite good agreement is reached for the values of the resonant frequencies as well as for the roll-off.

Numerical tests were made with the two different damping laws already discussed in 2.2.5. There was some influence on the relative height of the gravest resonant peak as the number of stages was increased: they were of equal height for the 'imaginary spring constant' case, but dropped with rising $n$ under the 'velocity-proportional' law. 


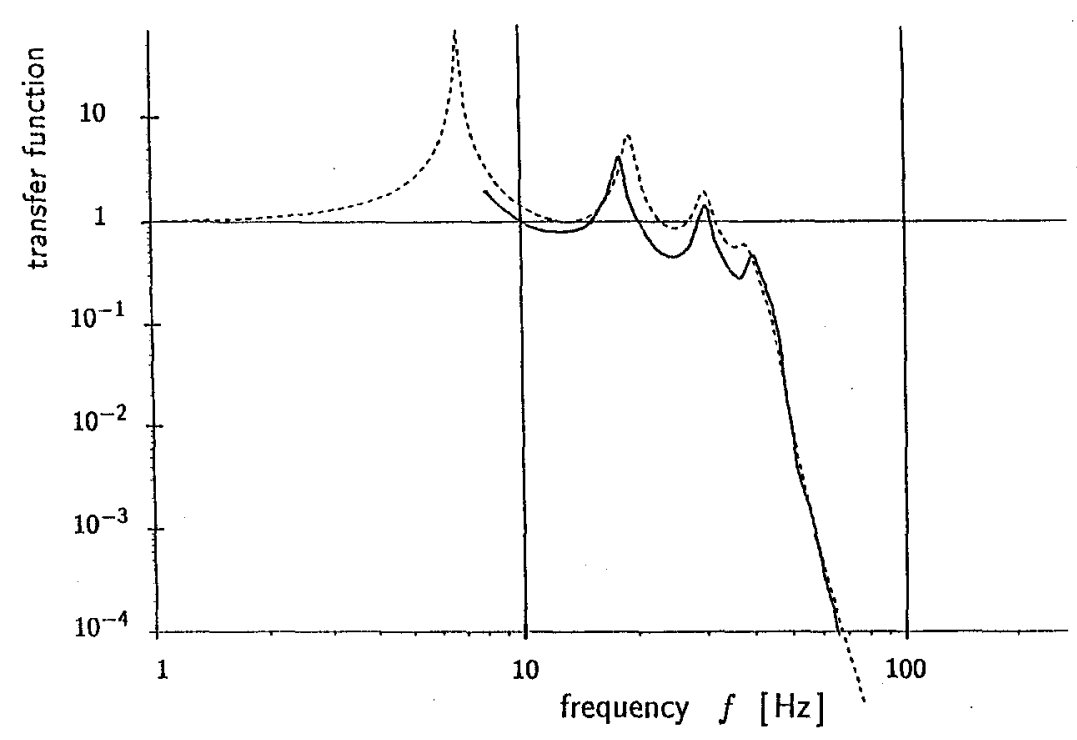

Fig. 5. Transmissibility (for vertical motion) of 4-stage RAL stack: RAL measurements (solid line) and MPQ model calculations (dashed line). Transmissibility is down to $10^{-4}$ at about $70 \mathrm{~Hz}$.

The influence of the damping law on the roll-off was not substantial in the frequency range of Figure 5, the superior suppression of the 'imaginary spring constant' case would become manifest only at somewhat higher frequencies.

\subsubsection{Garching stack measurements}

A set of four RAL stacks was used in one Garching end tank to support the top frame used for pendulum suspension. The four bottom plates supporting the four stacks at each corner can be driven (with a swept sine) in horizontal or vertical direction by four low-voltage piezo vibrators (PI P-844.20).

Piezoelectric accelerometers measured the spectra of the horizontal motion at the top frame and at the piezo-driven bottom plate. The quotient of these values gives the transmissibility. Typical measurements are presented in Figures 6 and 7, in both cases for horizontal motion.

The seismic isolation even of a stack having only three stages, as shown in Figure 6, was found fully sufficient for the present Garching 30-m prototype. Therefore it was decided to use them in the current upgrade in all three tanks. 


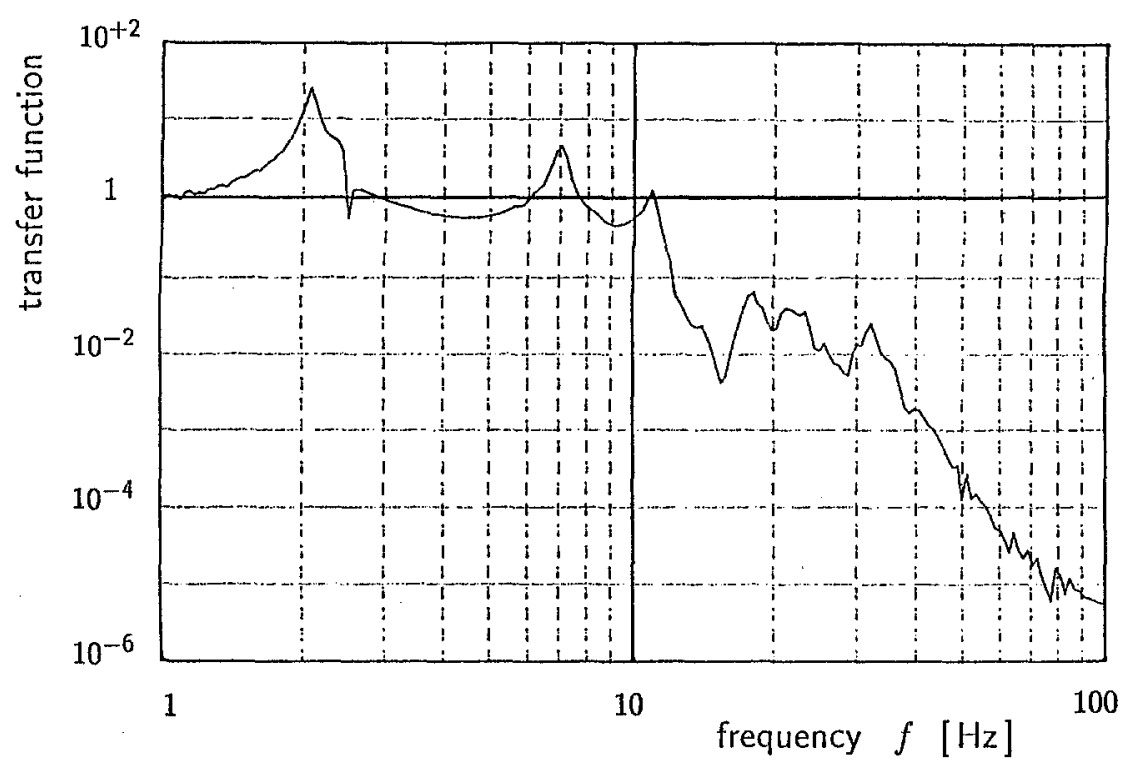

Fig. 6. Horizontal transmissibility of 3-stage RAL stack at Garching, in the frequency range from 1 to $100 \mathrm{~Hz}$. The resonant frequencies are near 2,8 , and $11 \mathrm{~Hz}$. The heavy horizontal line marks unity transfer function, the spacing of the horizontal lines is a factor of 10 each, the transmissibility is down to $10^{-5}$ at about $80 \mathrm{~Hz}$.

\subsubsection{Acoustic bypass}

Figure 7 shows the frequency range from the lowest resonance (near $2 \mathrm{~Hz}$ ) up to about $100 \mathrm{~Hz}$. The observed data (resonant frequencies, roll-off) were in good agreement with the expected behaviour, and they nourished the hope that the roll-off would continue to higher frequencies. This was not the case, but rather a resurging curve (shown in Figure 7, upper curve) was observed, rising almost towards unity again at $1 \mathrm{kHz}$.

This behaviour is difficult to explain from the elastic properties of the rubber springs, and some observations pointed to acoustic coupling through the air. The measurements were repeated in vacuum. This took some experimental effort: preamplifiers for operation in vacuum and avoiding crosstalk between the feedthroughs and leads of the high-power piezo drives and the (low-voltage) accelerometer signals.

The results were convincing: with the tank evacuated, the rise at frequencies above $100 \mathrm{~Hz}$ disappeared. This is shown in the lower curve of Figure 7. Intuitively, one would not have expected the heavy lead bricks to be so strongly excited by acoustics in the air.

But still the results were not fully satisfactory, as the transfer function seemed to level off at something like $10^{-6}$ at best, rather than continuing the steep roll-off. This behaviour is not yet fully understood, but the stack investigations had to be broken off for the moment as all three vacuum tanks 


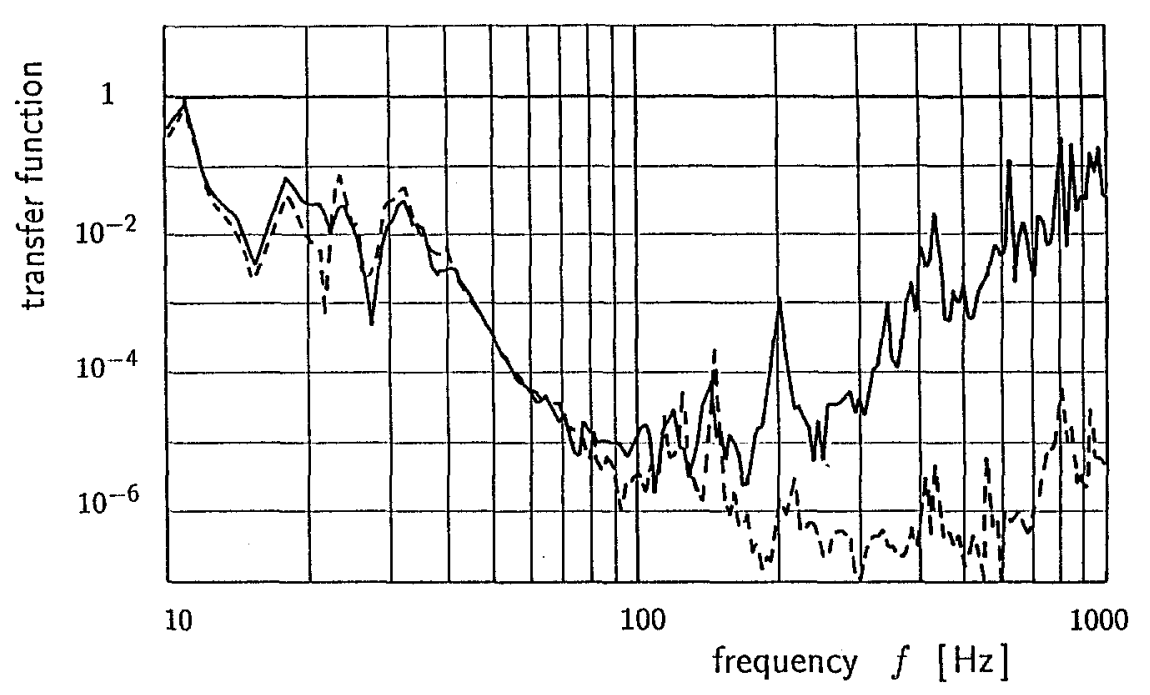

Fig. 7. Horizontal transmissibility of 3-stage RAL stack at Garching, in the range from $10 \mathrm{~Hz}$ to $1 \mathrm{kHz}$, measured in air (upper curve) and in vacuum (lower curve). Isolation is down to $10^{-4}$ at about $60 \mathrm{~Hz}$, and in vacuum reaches $10^{-6}$ in a frequency range from 150 to $500 \mathrm{~Hz}$.

are now used for installing an upgrade of the $30-\mathrm{m}$ prototype. It is hoped that the stack tests will be resumed soon, in a dedicated test tank.

The results of the stack measurements, although incomplete, were quite encouraging. Suppression factors of $10^{-5}$ and even $10^{-6}$ are readily achieved even with a three-stage stack. Inside the frequency range up to $200 \mathrm{~Hz}$, a further improvement with the number of stages can be predicted, the GEO specifications can no doubt be readily met.

One further important feature of stacks is that they can easily be made to have vertical isolation of similar quality as the horizontal one; or an even better one if one wants to make up for the shortcoming of the wire pendulum.

At higher frequencies it will yet have to be determined whether the measured levelling off in the transfer function is a physical effect of the stack structure, or whether it is an artefact in the very delicate measurement. But in any case, the specifications are not at risk, as the ground motion according to (17) rolls off with $f^{-2}$. 


\subsection{Ultra-low frequencies}

\subsubsection{Seismic noise at ultra-low frequencies}

There are various causes for very slow drifts in the distance between points as far apart as our arm lengths (i.e. $3 \mathrm{~km}$ ). Most prominent examples are the lunar and solar tides of the solid earth, but there are also seasonal and meteorological fluctuations.

Above frequencies $f_{\ell}=v_{s} / \ell$, determined by the velocity of sound $v_{s}$ in the ground and the arm length $\ell$, the motions at the two end points can be regarded as uncorrelated. This frequency $f_{l}$ is of the order of $1 \mathrm{~Hz}$. For drifts at much lower frequencies, the relative motion $\delta \ell$ is given by the low-frequency strain in the ground multiplied by the arm length.

These slow drifts are not inside the frequency range of measurement, but their effects need to be suppressed nevertheless. This is so because their relatively large swings could drive the interferometer far out of its proper point of operation.

For an example, let us consider the diurnal tides of the solid earth. Although the strains are quite small, in the order of a few 'nanostrain', i.e. strains of a few $10^{-9}$, this is, after all, twelve to thirteen powers of 10 larger than the gravity-wave signals we want to measure. The frequency, on the other hand, is only some six to eight powers of ten lower than our GW signal frequencies.

\subsubsection{Dynamic range of control elements}

Large low-frequency swings have grave consequences for the application of control signals. These have to do with the finite "dynamic range" of such control elements.

The first limitation (and the most fundamental one) is due to the electronic noise in the control amplifiers, say the current drivers for the coiland-magnet control elements.

Typically, low-noise amplifiers have a noise current that is at best ten powers of 10 below the full current. A control element designed to compensate drifts up to $1 \mathrm{~mm}$ will thus introduce a displacement noise in the order of $10^{-13} \mathrm{~m}$ at low frequencies, and even including the reduction of this noise due to the inertial mass of the mirror, this will still be several powers of 10 above the noise allowed when we want to measure strains of $h=10^{-21}$ or better.

An important consequence of this is that the control signals compensating the slow large drifts are not allowed to be applied to the mirrors themselves, but only to a stage higher up, say to the intermediate mass in a double pendulum system. A control system along these lines is just being investigated at Garching.

A second limitation arises because the coils of the coil-and-magnet systems are mounted to masses that in themselves are not totally quiet, that 
in some cases will have the full seismic noise of the ground. The coil-andmagnet systems can be operated such that the force exerted on the magnet is in first order independent of the position of the coil. But in the case of large low-frequency swings, this optimal point of operation cannot be maintained. Recent investigations have led to configurations of the coils in which also the second derivative of force with position vanishes. Such coils allow much larger swings, and they will be incorporated into the current upgrade of the Garching prototype.

\section{Conclusion}

It has been demonstrated that mechanical noise of various kinds can impose serious limitations on the sensitivity of interferometric gravitational wave detectors. In particular, the discussion has shown that in order to cope with these the arm length has to be chosen on the order of a few kilometers.

But a large arm length alone is no guarantee for success. Each of the noise sources discussed will require special attention and will call for an optimal design of the relevant parts of the detector.

In experiments on various prototypes the world over, and in detailed design studies, the feasibility of a gravitational wave detector with strain sensitivities of $10^{-21}$ or even $10^{-22}$ has been demonstrated. This is why applications for funding of the large detectors can no longer be considered premature, they reflect the high state of the art already achieved.

\section{References}

1. G. Schäfer: Gravity-Wave Astrophysics, Springer Lecture Notes in Physics, this issue.

2. K. Danzmann et al:: The GEO-Project: A Long-Baseline Laser Interferometer for the Detection of Gravitational Waves, Springer Lecture Notes in Physics, this issue.

3. W. Winkler et al:: The Optics of an Interferometric Gravitational-Wave Antenna, Springer Lecture Notes in Physics, this issue.

4. R. Mönchmeyer, G. Schäfer, E. Müller, and R.E. Kates, Astron. Astrophys. 246 (1991) 417.

5. Proposal for a Joint German-British Interferometric Gravitational Wave Detector, J. Hough, B. J. Meers, G. P. Newton, N. A. Robertson, H. Ward, G. Leuchs, T. M. Niebauer, A. Rüdiger, R. Schilling, L. Schnupp, H. Walther, W. Winkler, B. F. Schutz, J. Ehlers, P. Kafka, G. Schäfer, M. W. Hamilton, I. Schütz, H. Welling, J. R. J. Bennett, I. F. Corbett, B. W. H. Edwards, R.J.S. Greenhalgh, and V. Kose, Max-Planck-Institut für Quantenoptik Report No. MPQ 147 (1989).

6. R. Vogt, R.W.P. Drever, F.J. Raab, K.S. Thorne, and R. Weiss: Laser interferometer gravitational-wave observatory, Proposal to the NSF, 1989. 
7. A. Giazotto, A. Brillet, et al., The VIRGO Project, Proposal to INFN, 1989.

8. R.J. Sandeman, D.G. Blair, and J. Collett, Australian International Gravitational Research Centre, proposal to the Australian Government, (Australian National University, 1991).

9. N. Kawashima, Proc. Sixth Marcel Grossmann Meeting, Kyoto, 1991.

10. B.J. Meers, Phys. Rev. D 38, 2317 (1988); K.A. Strain and B.J. Meers, Phys. Rev. Lett. 66, 1391 (1991).

11. C.M. Caves, Phys. Rev. D 23, 1693 (1981).

12. W. Martin, Ph.D. Thesis, Glasgow (1978).

13. P.R. Saulson, Phys. Rev. D 42, 2437 (1991).

14. N.A. Robertson: Seismic Isolation, in: The Detection of Gravitational Radiation, Ed. D. Blair, Cambridge University Press, 1991.

15. M. Steinwachs, Geol. Jahrbuch E 3 (1974) 1-59.

16. A. Brüge and G. Lauer, PTB report PTB-MA-22 (1992).

17. D. Shoemaker, R. Schilling, L. Schnupp, W. Winkler, K. Maischberger, and A. Rüdiger, Phys. Rev. D 38, 423 (1988).

18. H. Billing, K. Maischberger, A. Rüdiger, R. Schilling, L. Schnupp, and W. Winkler, J. Phys. E: Sci. Instrum. 12 (1979) 1043-1050.

\section{List of Authors}

A. Rüdiger, J. Chen, K. Danzmann, P.G. Nelson, T.M. Niebauer, R. Schilling, K.A. Strain, L. Schnupp, H. Walther, W. Winkler, Max-Planck-Institut für Quantenoptik, D-8046 Garching, Germany; J. Hough, A.M. Campbell, C.A. Cantley, J.E. Logan, B.J. Meers, E. Morrison, G.P. Newton, D.I. Robertson, N.A. Robertson, S. Rowan, K.D. Skeldon, P.J. Veitch, and H. Ward, Department of Physics and Astronomy, University of Glasgow, Glasgow, UK; H. Welling, P. Aufmuth, I. Kröpke and D. Ristau, Laser-Zentrum and Institut für Quantenoptik, Universität Hannover, D-3000 Hannover, Germany; J.E. Hall, J.R.J. Bennett, I.F. Corbett, B.W.H. Edwards, R.J. Elsey, and R.J.S. Greenhalgh, Rutherford Appleton Laboratory, Chilton, Didcot, UK; B.F. Schutz, D. Nicholson, and J.R. Shuttleworth, Department of Physics, University of Wales, Cardiff, UK; J. Ehlers, P. Kafka, and G. Schäfer, Max-Planck-Institut für Astrophysik, D-8046 Garching, Germany; H. Braun, Bauabteilung der Max-Planck-Gesellschaft, D-8000 München, Germany; V. Kose, Physikalisch-Technische Bundesanstalt, D. 3300 Braunschweig and D-1000 Berlin, Germany. 